\title{
BUDD-CHIARI SYNDROME AS INITIAL MANIFESTATION OF BEHÇET'S DISEASE
}

Camille Constanza Codogno Postigo Castro ${ }^{1, \star}$, Vani Abreu de Souza Filho ${ }^{1}$, Flávia Carolina Oliveira ${ }^{1}$, Daniel Ferraz Campos Mazo $^{1}$, Heloisa Sisconeto Bisinotto ${ }^{1}$, Carla Jerônimo Peres Fingerhut ${ }^{1}$, Alisson Alien Vigano Pugliesi ${ }^{1}$, Zoraida Sachetto ${ }^{1}$

1.Universidade Estadual de Campinas, Campinas (SP), Brazil.

*Corresponding author: camille_postigo@yahoo.com.br

\section{BACKGROUND}

Budd-Chiari syndrome (BCS) is characterized by obstruction of hepatic venous drainage, usually by thrombosis in hepatic veins or inferior vena cava. The main causes of BCS are diseases associated with hypercoagulation states and myeloproliferative conditions, but BCS can be a rare vascular manifestation of Behçet disease (BD). In a study at the Faculty of Medicine in Eskişehir Osmangazi University, Turkey, BCS was observed in 4 of 300 patients with BD (1.3\%). The low prevalence is confirmed in other surveys, which demonstrates the relevance of this case report.

\section{CASE REPORT}

A 33-year-old male patient with no history of liver cirrhosis, developed wasting syndrome and ascites with a need for regular paracentesis. Abdominal computed tomography (CT) was consistent with thrombosis filling the middle and left hepatic veins and part of the right hepatic vein, extending to the inferior vena cava (Fig. 1). A diagnosis of BCS was made, manifesting with posthepatic hypertension. At the time, the presence of coagulopathies was investigated. Tested positive in a single sample for lupus anticoagulant. During the investigation, colonoscopy was performed, demonstrating multiple aphthoid erosions and isolated ulcer in the terminal ileum. Inflammatory bowel disease was ruled out by the gastroenterology staff. Even in full anticoagulation, it was necessary to place a stent in the inferior vena cava. On rheumatology evaluation, the patient reported recurrent oral ulcers since the diagnosis of BCS. Thus, the hypothesis of BCS secondary to BD was raised. Given the severity of the condition, monthly intravenous cyclophosphamide therapy was initiated. After two cycles of cyclophosphamide, the patient presented enterorrhagia. Erosions in terminal ileum continued to be seen at colonoscopy and scintigraphic signs of bleeding in the ileocecal region were shown in SPECT/ CT. At the time, a $1 \mathrm{~cm}$ ulcerated lesion was observed in the perianal region. Bleeding ileocecal ulcerations were then considered as indicative of disease activity, and intravenous methylprednisolone and maintenance of monthly cyclophosphamide were indicated.
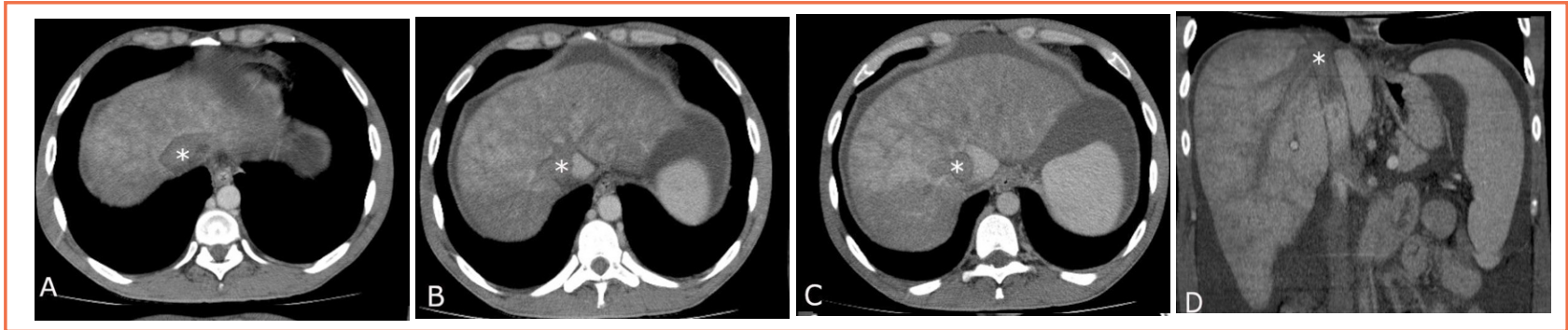

Figure 1. Computed tomography in serial axial (A, B, C) and coronal (D) sections, in the portal phase after injection of the intravenous contrast showing extensive failure to fill the inferior vena cava and hepatic veins, compatible with thrombosis $\left({ }^{*}\right)$, associated with perfusion disorder of the hepatic parenchyma and massive ascites

\section{CONCLUSION}

The case presented reports a rare but severe manifestation of BD. Rheumatologists and gastroenterologists must work together on these patients, considering the treatment is not usual to BCS. 